\title{
Influência do uso de protetores bucais sobre o consumo de oxigênio: artigo de revisão
}

\author{
Influence of the use of mouth guards on oxygen consumption: review article
}

\begin{abstract}
Bárbara Capitanio de Souza ${ }^{1}$
${ }^{1}$ Academia Brasileira de Odontologia do Esporte, Rio de Janeiro, RJ, Brasil

- A autora declara que não há conflito de interesse.
\end{abstract}

\section{Resumo}

Objetivo: avaliar, através da literatura, se o uso de protetores bucais influencia os valores de $\mathrm{VO}_{2}$ e $\mathrm{VO}_{2}$ máx. durante uma atividade física. Material e Métodos: o estudo foi realizado em janeiro de 2017 e incluiu os artigos indexados na base de dados MEDLINE / PubMed, desde o ano 2000 até o momento da pesquisa. A estratégia de busca empregou o termo protetor bucal com as seguintes palavras e possíveis combinações: limiares ventilatórios, desempenho atlético, volume máximo de oxigênio, capacidade de desempenho aeróbio e consumo de oxigênio. Os trabalhos encontrados foram apresentados em uma tabela e analisados descritivamente. Resultados: os dados analisados indicam que o uso do protetor bucal Tipo III ou personalizado não influencia negativamente os valores VO 2 $\mathrm{VO}_{2}$ máx. Conclusão: de acordo com a literatura, os protetores bucais Tipo I e Tipo Il tiveram influência negativa nas variáveis analisadas. 0 uso do protetor bucal personalizado, especialmente do Tipo III, não apresentou qualquer dano aos valores $\mathrm{VO}_{2}$ e $\mathrm{VO}_{2}$ máx.

Palavras-chave: Protetores bucais; Desempenho atlético; Consumo de oxigênio.

\section{ABSTRACT}

Objective: Objective: to evaluate, through a literature review, whether the use of mouth guards influences the $\mathrm{VO}_{2}$ and $\mathrm{VO}_{2}$ max values during physical activity. Material and Methods: the study was carried out in January 2017 and included the articles indexed in the MEDLINE/PubMed database from the year 2000 until the date of the search. The search strategy employed the term "mouth guard" with the following words and possible combinations: "ventilatory thresholds," "athletic performance," "maximum volume of oxygen," "aerobic performance capacity," and "oxygen consumption." The articles found were presented in a table and analyzed descriptively. Results: the data analyzed indicate that the use of the Type III or customized mouth guard does not negatively influence the $\mathrm{VO}_{2}$ and $\mathrm{VO}_{2}$ max values. Conclusion: according to the literature, Type I and Type II mouth guards had a negative influence on the analyzed variables. The use of the personalized mouth guard, especially Type III, did not present any damage to the $\mathrm{VO}_{2}$ and $\mathrm{VO}_{2}$ max values.

Keywords: Mouth protectors; Athletic performance; Oxygen consumption.

\section{Introdução}

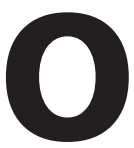
atleta, durante a realização das suas atividades esportivas, fica suscetível a inúmeras injúrias. $\mathrm{O}$ uso de protetor bucal $(\mathrm{PB})$ pode fornecer proteção aos dentes, à mandíbula, aos tecidos moles adjacentes e, também, proteger o cérebro contra concussão decorrentes dos impactos na região de mandíbula. ${ }^{1} \mathrm{O}$ uso de protetores mal adaptados e pouco retentivos ocasionaram dificuldades de fala e de respiração, sensação de ânsia, dor ou ferimentos, além de modificar negativamente o volume da ventilação, durante a atividade física, podendo, com isso, alterar alguns parâmetros fisiológicos. ${ }^{2}$

Dentre os parâmetros fisiológicos utilizados na investigação da avaliação funcional de atletas, podemos citar o consumo de oxigênio $\left(\mathrm{VO}_{2}\right)$, que é um indicador de funcionalidade e reflete a exigência energética durante uma atividade física, e o consumo máximo de oxigênio $\left(\mathrm{VO}_{2}\right.$ máx), que auxilia na avaliação do desempenho físico e da capacidade funcional cardiorrespiratória. $\mathrm{O} \mathrm{VO}_{2}$ máx. é considerado um preditor de desempenho, pois a capacidade do ser humano para realizar exercícios de longa e média duração depende principalmente do metabolismo aeróbio. Os valores de $\mathrm{VO}_{2}$ e $\mathrm{VO}_{2}$ máx. refletem a con- dição física do indivíduo e são utilizados para avaliar e comparar o desempenho de atletas. ${ }^{3}$ Sendo assim, o uso de qualquer acessório que dificulte esta captação de oxigênio é indesejável.

Os protetores bucais foram desenvolvidos para reduzir possíveis danos e/ou injúrias decorrentes da prática esportiva, contudo muitos atletas preferem não usá-los devido à crença nos efeitos negativos sobre a respiração, durante a atividade física. Por isso, o objetivo nesse trabalho é avaliar, através da literatura, se o uso de protetores bucais influencia os valores de $\mathrm{VO}_{2}$ e $\mathrm{VO}_{2}$ máx. durante uma atividade física.

\section{Material e Métodos}

Trata-se de um estudo descritivo analítico, que foi realizado através de revisão de literatura. A estratégia de busca empregou o termo protetor bucal (mouthguard) com o operador booleano and e os descritores: limiares ventilatórios (ventilatory thresholds), desempenho atlético (Athletic performance), volume máximo de oxigênio (maximum volume of oxygen), capacidade de desempenho aeróbio (aerobic performance capacity) e consumo de oxigênio (oxygen consumption). Para uma leitura mais atual, 
foram selecionados os artigos publicados entre o ano 2000 até janeiro de 2017. Para uma análise inicial, foram excluídos os artigos que não apresentavam resumo disponível. Após a avaliação dos resumos, foram excluídos os artigos que não tratavam do tema proposto, que realizaram experimentos em animais, que reportavam relatos de caso e os artigos duplicados. A busca inicial identificou 91 artigos indexados, sendo que 10 foram selecionados, de acordo com os critérios expostos, para compor a revisão final (Figura 1). Os dados foram apresentados em uma tabela e analisados descritivamente.

\section{Base de dados MEDLINEIPubmed}

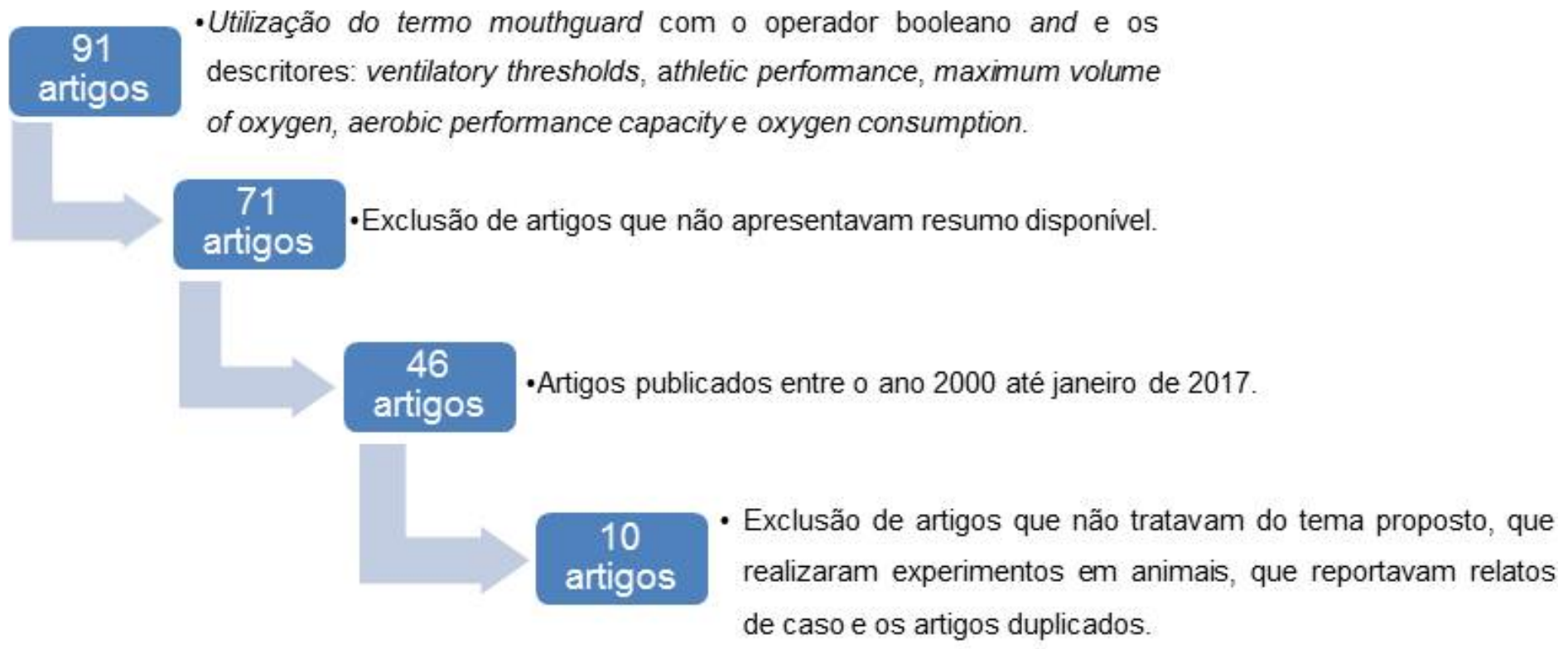

Figura 1. Fluxo de busca dos artigos para compor a pesquisa

\section{Revisão de Literatura}

A Odontologia, como área de atuação no esporte, ainda é pouco pesquisada. Ao analisarmos a literatura disponível, encontramos uma relativa escassez de estudos sobre assuntos mais específicos, que fogem da abordagem tradicional sobre os traumatismos, durante a prática esportiva. São poucos os estudos disponíveis que analisam outros aspectos relacionados aos protetores bucais, como as variáveis ventilatórias. Com o crescente desenvolvimento científico e interesse pela área da Odontologia do Esporte, novas pesquisas estão sendo desenvolvidas para a firmação do conhecimento na especialidade.

Alguns parâmetros fisiológicos são importantes para identificar a capacidade funcional do atleta. Os valores de $\mathrm{VO}_{2}$ e $\mathrm{VO}_{2}$ máx. têm recebido a atenção de vários pesquisadores fisiologistas, pois influenciam no desempenho físico do esportista. Assim, existe uma preocupação com a alteração do volume de ventilação, quando se utiliza algum tipo de PB. Os estudos selecionados para a revisão, descritos na tabela 1, tentaram identificar, através de testes físicos, a existência de alterações nos valores de parâmetros fisiológicos ventilatórios, quando utilizado o PB. Nesta revisão, foi dada maior ênfase aos valores de $\mathrm{VO}_{2} \mathrm{eVO}_{2}$ máx. De modo geral, nestes estudos, foram citados três diferentes tipos de dispositivos de proteção, para suas experimentações (Tabela 2). 
Tabela 1. Resumo descritivo das principais características dos artigos selecionados

\begin{tabular}{|c|c|c|c|c|c|}
\hline Estudos & $\begin{array}{l}\text { Atividade } \\
\text { Física }\end{array}$ & $\begin{array}{c}\text { Protetor } \\
\text { Analisado }\end{array}$ & $\begin{array}{c}\text { Variáveis } \\
\text { Analisadas }\end{array}$ & Desfecho & Limitações e Viesses \\
\hline $\begin{array}{c}\text { Keçeci et al. }{ }^{4} \\
2005\end{array}$ & $\begin{array}{l}\text { Teste } \\
\text { shuttle-run }\end{array}$ & Tipo III & VO2 máx, & $\begin{array}{l}\text { O PB não influenciou negativamente as variá- } \\
\text { veis analisadas, não havendo efeito significati- } \\
\text { vo em comparação ao não uso de PB. }\end{array}$ & $\begin{array}{l}\text { Não foi realizado teste em esteira } \\
\text { ergométrica. } \\
\text { Os parâmetros ventilatórios foram } \\
\text { medidos através de um aparelho } \\
\text { portátil. }\end{array}$ \\
\hline $\begin{array}{l}\text { Delaney et al. } .^{5} \\
\quad 2005\end{array}$ & $\begin{array}{l}\text { Esteira de } \\
\text { patinação }\end{array}$ & Tipo II & $\mathrm{VO}_{2}$ & $\begin{array}{l}\text { Foi observada uma influência negativa sobre } \\
\text { as variáveis analisadas, havendo efeito signifi- } \\
\text { cativo em comparação ao não uso de PB. }\end{array}$ & $\begin{array}{l}\text { Não foi realizado teste em esteira } \\
\text { ergométrica. } \\
\begin{array}{c}\text { Limitação quanto ao tamanho } \\
\text { amostral. }\end{array}\end{array}$ \\
\hline $\begin{array}{l}\text { Bourdin et al. }{ }^{6} \\
\quad 2006\end{array}$ & $\begin{array}{c}\text { Esteira } \\
\text { ergométrica }\end{array}$ & $\begin{array}{l}\text { Tipo II } \\
\text { Tipo III }\end{array}$ & $\begin{array}{c}\mathrm{VO}_{2} \\
\mathrm{VO}_{2} \text { máx }\end{array}$ & $\begin{array}{c}\text { Não foi identificada interferência sobre os } \\
\text { parâmetros fisiológicos analisados, para } \\
\text { ambos PB. }\end{array}$ & $\begin{array}{l}\text { Limitação quanto à padronização e } \\
\text { ao tamanho amostral. } \\
\text { Não esclarece se houve algum tipo } \\
\text { de ajuste adicional no PB Tipo II } \\
\text { realizado pelo cirurgião-dentista. }\end{array}$ \\
\hline $\begin{array}{l}\text { Von Arx et al. }{ }^{7} \\
2008\end{array}$ & $\begin{array}{l}\text { Bicicleta } \\
\text { ergométrica }\end{array}$ & Tipo III & $\mathrm{VO}_{2}$ & $\begin{array}{l}\text { O PB não influenciou os parâmetros anali- } \\
\text { sados, não havendo efeito significativo em } \\
\text { comparação ao não uso de PB. }\end{array}$ & $\begin{array}{l}\text { Limitação quanto à padronização e } \\
\text { ao tamanho amostral. }\end{array}$ \\
\hline $\begin{array}{c}\text { Gebauer, et al. }{ }^{8} \\
2011\end{array}$ & $\begin{array}{c}\text { Esteira } \\
\text { ergométrica }\end{array}$ & Tipo III & $\mathrm{VO}_{2}$ & $\begin{array}{l}\text { Não houve influência negativa sobre as variá- } \\
\text { veis analisadas, não havendo efeito significati- } \\
\text { vo em comparação ao não uso de PB. }\end{array}$ & $\begin{array}{l}\text { Limitação quanto à padronização e } \\
\text { ao tamanho amostral. }\end{array}$ \\
\hline $\begin{array}{l}\text { Gamer, et al. }{ }^{9} \\
2011\end{array}$ & $\begin{array}{c}\text { Teste } \\
\text { steady-state }\end{array}$ & Tipo III & $\mathrm{VO}_{2}$ & $\begin{array}{l}\text { O PB influenciou na melhora das variáveis } \\
\text { analisadas, havendo efeito positivo significati- } \\
\text { vo em comparação ao não uso de protetor. }\end{array}$ & $\begin{array}{l}\text { Não foi realizado teste em esteira } \\
\text { ergométrica. } \\
\text { Limitação quanto à padronização e } \\
\text { ao tamanho amostral. }\end{array}$ \\
\hline $\begin{array}{l}\text { Rapisura et al. }{ }^{10} \\
2012\end{array}$ & $\begin{array}{l}\text { Bicicleta } \\
\text { ergométrica }\end{array}$ & $\begin{array}{l}\text { Tipo II } \\
\text { Tipo III }\end{array}$ & $\mathrm{VO}_{2}$ & $\begin{array}{l}\text { O PB não influenciou negativamente as variá- } \\
\text { veis analisadas, não havendo efeito significati- } \\
\text { vo em comparação ao não uso de PB. }\end{array}$ & $\begin{array}{l}\text { Limitação quanto ao tamanho } \\
\text { amostral. }\end{array}$ \\
\hline $\begin{array}{l}\text { Queiróz et al. }{ }^{11} \\
2013\end{array}$ & $\begin{array}{l}\text { Teste } \\
\text { shuttle-run } \\
\text { e teste de } \\
\text { Cooper }\end{array}$ & $\begin{array}{l}\text { Tipo I } \\
\text { Tipo II } \\
\text { Tipo III }\end{array}$ & $\mathrm{VO}_{2}$ máx & $\begin{array}{c}\text { O uso dos PB Tipo I e Tipo II teve uma influ- } \\
\text { ência negativa sobre as variáveis analisadas, } \\
\text { havendo efeito significativo em relação ao } \\
\text { não uso de PB. } \\
\text { O PB Tipo III influenciou na melhora das } \\
\text { variáveis analisadas, havendo efeito positivo } \\
\text { significativo em comparação ao não uso de } \\
\text { protetor. }\end{array}$ & $\begin{array}{l}\text { Não foi realizado teste em esteira } \\
\text { ergométrica. }\end{array}$ \\
\hline $\begin{array}{c}\text { Collares et al. }{ }^{12} \\
2013\end{array}$ & $\begin{array}{l}\text { Teste de } \\
\text { shuttle-run }\end{array}$ & Tipo III & $\mathrm{VO}_{2}$ máx & $\begin{array}{l}\text { O PB não influenciou negativamente as variá- } \\
\text { veis analisadas, não havendo efeito significati- } \\
\text { vo em comparação ao não uso de PB. }\end{array}$ & $\begin{array}{l}\text { Não foi realizado teste em esteira } \\
\text { ergométrica. }\end{array}$ \\
\hline $\begin{array}{l}\text { Pierro et al. }{ }^{13} \\
\quad 2015\end{array}$ & $\begin{array}{c}\text { Bicicleta } \\
\text { ergométrica }\end{array}$ & Tipo III & $\mathrm{VO}_{2}$ & $\begin{array}{l}\text { O PB influenciou na melhora das variáveis } \\
\text { analisadas, havendo efeito positivo significati- } \\
\text { vo em comparação ao não uso de protetor. }\end{array}$ & $\begin{array}{l}\text { Limitação quanto ao tamanho } \\
\text { amostral. }\end{array}$ \\
\hline
\end{tabular}

Legenda: $\mathrm{VO}_{2}$ máx.: consumo máximo de oxigênio

$\mathrm{VO}_{2}$ : consumo de oxigênio

Tabela 2. Tipos de protetores bucais utilizados nos estudos

\begin{tabular}{|c|c|c|c|c|}
\hline \multirow[b]{2}{*}{ Tipo } & \multirow[b]{2}{*}{ Característica } & & & \\
\hline & & Retenção & Proteção Efetiva & Custo \\
\hline $\begin{array}{c}\text { Tipo I: Pré-fabricado ou } \\
\text { de estoque }\end{array}$ & $\begin{array}{c}\text { Possui três tamanhos padronizados: pequeno, médio e } \\
\text { grande. Pode interferir na fala e na respiração normal. Seu } \\
\text { uso é pouco indicado. Não permite adaptação personalizada } \\
\text { na arcada dentária. }\end{array}$ & Baixa & Baixa & Baixo \\
\hline Tipo II: Termoplástico & $\begin{array}{c}\text { Permitem uma adaptação um pouco melhor do que o Tipo } \\
\text { I. É necessário aquecimento em água para a modelagem do } \\
\text { protetor. }\end{array}$ & Moderada & Moderada & $\begin{array}{l}\text { Baixo a } \\
\text { moderado }\end{array}$ \\
\hline $\begin{array}{l}\text { Tipo III: Individual e feito } \\
\text { sobre medida }\end{array}$ & $\begin{array}{c}\text { Muito superior aos PB do Tipo I e II em termos de retenção, } \\
\text { adaptação e proteção. São mais confortáveis e interferem } \\
\text { menos na respiração e na fala. Fabricado sobre um modelo } \\
\text { vazado em gesso, usando material termoplástico, em apare- } \\
\text { Iho a vácuo. É o modelo mais indicado. }\end{array}$ & Alta & Alta & Alto \\
\hline
\end{tabular}


De modo geral, o uso dos protetores do Tipo I e II teve uma influência negativa sobre as variáveis analisadas, havendo efeito significativo em relação ao não uso de $\mathrm{PB}$, indicando prejuízo sobre os limiares ventilatórios. ${ }^{5,11} \mathrm{Ou}-$ tros estudos que avaliaram protetores do Tipo II e III não identificaram interferência sobre os parâmetros ventilatórios analisados, apresentando resultados semelhantes de $\mathrm{VO}_{2}$ e $\mathrm{VO}_{2}$ máx. com ou sem o uso de PB. ${ }^{4,6-8,10,12}$ Contudo, em algumas pesquisas, o PB Tipo III influenciou na melhora das variáveis ventilatórias analisadas, havendo efeito positivo significativo em comparação ao não uso de protetor, sugerindo maiores benefícios para o atleta..$^{9,11,13}$

\section{Discussão}

Os protetores bucais possuem diferentes funções como proteger as estruturas dentais e periodontais, durante a prática esportiva, e amenizar o número e a gravidade dos traumatismos. ${ }^{1}$ Os trabalhos envolvendo protetores bucais já são realizados há algumas décadas; contudo, é recente a investigação sobre a influência dos protetores sobre os parâmetros ventilatórios, o que gera dúvidas para pacientes e profissionais. Entretanto, os avanços tecnológicos e científicos relacionados com as técnicas de confecção e com o desenvolvimento de novos materiais, associados à crescente competitividade no meio esportivo, estimularam o surgimento de novas pesquisas, que abordam a relação entre o uso de protetores bucais e o desempenho esportivo.

Para melhor esclarecer a importância do conhecimento sobre a existência de uma possível alteração dos parâmetros ventilatórios com o uso de protetores bucais é importante entendermos os significados desses. $\mathrm{O} \mathrm{VO}_{2}$ é uma medida da capacidade funcional do organismo em ofertar e utilizar o oxigênio para a produção de energia, aumentando linearmente com o trabalho muscular crescente. ${ }^{3} \mathrm{O} \mathrm{VO}_{2}$ máx. é a capacidade máxima do corpo de um indivíduo para transportar e utilizar oxigênio, durante o exercício incremental, o que reflete a condição física do indivíduo e é utilizado para avaliar e comparar o desempenho de atletas. $\mathrm{O} \mathrm{VO}_{2}$ máx. é um dos principais indicadores de aptidão funcional cardiorrespiratória. ${ }^{14}$ Assim, estes parâmetros são de grande importância para a avaliação funcional do atleta. ${ }^{3}$

Os trabalhos analisados indicam que, de acordo com o tipo de protetores bucais utilizados, pode haver modificação do desempenho esportivo, uma vez que as variáveis ventilatórias podem sofrer alteração. Os protetores bucais do Tipo I e Tipo II influenciaram negativamente as variáveis analisadas, havendo efeito significativo em compa- ração ao não uso de protetor. ${ }^{5,11}$ Alguns trabalhos indicaram que o PB Tipo II não influenciou negativamente as variáveis analisadas, não havendo efeito significativo em comparação ao não uso de protetor. ${ }^{6,10}$ Contudo, os autores não esclarecem se houve algum tipo de ajuste adicional realizado pelo cirurgião-dentista, nestes $\mathrm{PB}$. Isto poderia melhorar a adaptação do protetor e reduzir seus efeitos negativos sobre o desempenho. O PB Tipo III apresentou os melhores resultados, não influenciando negativamente as variáveis analisadas, havendo efeito significativo em comparação ao não uso de protetor. ${ }^{6,710,12}$ Além disso, alguns autores relataram que esse tipo de protetor influenciou positivamente os parâmetros avaliados, havendo efeito significativo favorável em comparação ao não uso de $\mathrm{PB} .{ }^{9,11,13}$ Isto significa que os atletas podem ter um benefício no desempenho com o uso do PB Tipo III.

Os estudos selecionados apresentaram algumas limitações. No que se refere ao universo e à amostra, alguns autores apresentaram um tamanho amostral reduzido de participantes, sem uma indicação cálculo amostral. Além disso, não há uma padronização dos participantes o que é importante levando em consideração os fatores fisiológicos. ${ }^{5-10,13}$ Outro fator importante a considerar é que algumas pesquisas não utilizaram o teste de ergoespirometria padrão para coleta de dados dos parâmetros ventilatórios. ${ }^{4,5,9,11,12} \mathrm{O}$ teste consiste em realizar um exercício progressivo em esteira ou bicicleta ergométrica, respirando por um dispositivo que permite a análise das variáveis ventilatórias com maior precisão.

A confecção do PB deve seguir as técnicas recomendadas, para contemplar a máxima eficácia do aparelho e o mínimo prejuízo para o atleta. Dentre estas orientações ressaltamos que o PB deve adaptar-se de forma justa ao arco dentário, recobrir as superfícies oclusais dos dentes, estender-se na região posterior, até a superfície distal do penúltimo dente presente no arco, para não provocar náuseas, devem estender-se além da linha muco-gengiva, vestibularmente. Além disso, é importante que a região palatal deva estender-se por, aproximadamente, $5 \mathrm{~mm}$ sobre o palato e conter sua extremidade fina, lisa e arredondada, para evitar interferências com a fala, a respiração ou estimular de reflexo de vômito. ${ }^{15}$ Os protetores bucais do Tipo I e Tipo II não contemplam as exigências mínimas para um protetor que deva ao mesmo tempo cumprir com os seus objetivos, sem prejudicar o desempenho do atleta. Além disso, é bastante frequente a queixa de desconforto pelo uso destes protetores, o que gera ideia equivocada e generalizada. ${ }^{2}$

Rev. Bras. Odontol., Rio de Janeiro, v. 74, n. 2, p. 150-4, abr./jun. 2017 


\section{Conclusão}

Os protetores Tipo I e Tipo II apresentaram uma influência negativa sobre as variáveis analisadas, havendo efeito significativo em relação ao não uso de $\mathrm{PB}$. A utilização do $\mathrm{PB}$ personalizado, especialmente o tipo III, não apre-

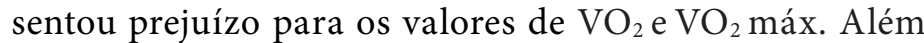
disso, alguns autores apontam uma melhora na capacidade anaeróbia, quando utilizado o protetor personalizado, o que pode indicar uma redução de níveis de lactato e fadiga muscular e melhora dos parâmetros ventilatórios. A confecção do $\mathrm{PB}$ deve seguir algumas recomendações, para contemplar a máxima eficácia do aparelho e o mínimo prejuízo para o atleta. É importante que essa confecção seja realizada pelo cirurgião-dentista com conhecimento sobre os dispositivos orais de proteção, para que se possa realizar a adequada indicação de uso.

\section{Referências}

1. Gould TE, Piland SG, Caswell SV, Ranalli D, Mills S, Ferrara MS, et al. National Athletic Trainers' Association Position Statement: Preventing and Managing Sport-Related Dental and Oral Injuries. J Athl Train. 2016;51(10):821-39.

2. Morales J, Buscà B, Solana-Tramunt M, Miró A. Acute effects of jaw clenching using a customized mouthguard on anaerobic ability and ventilatory flows. Hum Mov Sci. 2015;44:270-6.

3. Mainguy V, Malenfant S, Neyron AS, Saey D, Maltais F, Bonnet S, et al. Alternatives to the six-minute walk test in pulmonary arterial hypertension. PLoS One. 2014;9(8):e103626.

4. Keçeci AD, Cetin C, Eroglu E, Baydar ML. Do custom-made mouthguards have negative effects on aerobic performance capacity of athletes? Dent Traumatol. 2005;21(5):276-80.

5. Delaney JS, Montgomery DL. Effect of noncustom bimolar mouthguards on peak ventilation in ice hockey players. Clin J Sport Med. 2005;15(3):154-7.

6. Bourdin M, Brunet-Patru I, Hager PE, Allard Y, Hager JP, Lacour JR, et al. Influence of maxillary mouthguards on physiological parameters. Med Sci Sports Exerc. 2006;38(8):1500-4.

7. von Arx T, Flury R, Tschan J, Buergin W, Geiser T. Exercise capacity in athletes with mouthguards. Int J Sports Med. 2008;29(5):435-8.

8. Gebauer DP, Williamson RA, Wallman KE, Dawson BT. The effect of mouthguard design on respiratory function in athletes. Clin J Sport Med. 2011;21(2):95-100.
9. Garner DP, Dudgeon WD, Scheett TP, McDivitt EJ. The effects of mouthpiece use on gas exchange parameters during steady-state exercise in college-aged men and women. J Am Dent Assoc. 2011;142(9):1041-7.

10. Rapisura KP, Coburn JW, Brown LE, Kersey RD. Physiological variables and mouthguard use in women during exercise. J Strength Cond Res. 2010;24(5):1263-8. 11. Queiróz AF, de Brito RB Jr, Ramacciato JC, Motta RH, Flório FM. Influence of mouthguards on the physical performance of soccer players. Dent Traumatol. 2013;29(6):450-4.

12. Collares K, Correa MB, Mohnsam da Silva IC, Hallal PC, Demarco FF. Effect of wearing mouthguards on the physical performance of soccer and futsal players: a randomized cross-over study. Dent Traumatol. 2014;30(1):55-9.

13. Piero M, Simone U, Jonathan M, Maria S, Giulio G, Francesco T, Gabriella C, et al. Influence of a custom-made maxillary mouthguard on gas exchange parameters during incremental exercise in amateur road cyclists. J Strength Cond Res. 2015;29(3):672-7.

14. Wiewelhove T, Raeder C, Meyer T, Kellmann M, Pfeiffer M, Ferrauti A. Markers for Routine Assessment of Fatigue and Recovery in Male and Female Team Sport Athletes during High-Intensity Interval Training. PLoS One. 2015; 10(10):e0139801. 15. Gonçalves AR, Albuquerque HCL, Ferreira MCCF, e Souza CHC. Mouthguards: types and manufacturing technique. Prosthes Lab Sci. 2012;2(5):61-8.

\section{Mini Currículo e Contribuição dos Autores}

1. Bárbara Capitanio de Souza - cirurgiã-dentista e mestre.

Recebido em: 08/01/2017 / Aprovado em: 08/03/2017

Autora Correspondente

Bárbara Capitanio de Souza

E-mail: barbara.capitanio@gmail.com 